\title{
Elvitegravir/Cobicistat/Emtricitabine/Tenofovir Disoproxil Fumarate
}

\author{
National Cancer Institute
}

\section{Source}

National Cancer Institute. Elvitegravir/Cobicistat/Emtricitabine/Tenofovir Disoproxil

Fumarate. NCI Thesaurus. Code C157404.

\begin{abstract}
A fixed combination of elvitegravir, a human immunodeficiency virus type 1 (HIV-1) integrase strand transfer inhibitor (INSTI); cobicistat, a cytochrome P450 3A (CYP3A) inhibitor; emtricitabine, a nucleoside reverse transcriptase (RT) inhibitor (NRTI) analog of cytidine; and tenofovir disoproxil fumarate, an NRTI analog of adenosine monophosphate, that is used to treat HIV infection. Upon oral administration, elviteg ravir inhibits the activity of integrase, an HIV-1 coded enzyme that is necessary for viral replication. Inhibition of integrase prevents the integration of HIV-1 DNA into host genomic DNA. Cobicistat inhibits CYP3A, thereby limiting the oxidative metabolism of elvitegravir and increases its systemic exposure. Emtricitabine and tenofovir disoproxil fumarate inhibit the activity of HIV RT by competing with natural deoxyribonucleosides for incorporation into the growing viral DNA chain resulting in early chain termination. This interferes with the generation of DNA copies of viral RNA, which is necessary for the synthesis of new virions.
\end{abstract}

